\title{
Ultrasonic System Models for Pulse Trains Excitation Tuning
}

\author{
Linas Svilainis $^{1}$, Alberto Rodriguez ${ }^{2}$, Arturas Aleksandrovas ${ }^{1}$ \\ ${ }^{1}$ Department of Signal Processing, Kaunas University of Technology, \\ Studentu St. 48, LT-51367 Kaunas, Lithuania \\ ${ }^{2}$ Department of Communications Engineering, Universidad Miguel Hernandez, \\ Avda. Universidad S/N, 03203 Elche, Spain \\ linas.svilainis@ktu.lt
}

\begin{abstract}
Quality of the ultrasonic imaging depends on probing signal. Application of the spread spectrum (SS) signals offers significant advantage over conventional signals: higher signal-to-noise ratio can be achieved and spectral content can be programmed. Yet, such SS signals like chirp and coded sequences suffer for the sidelobes which are induced by compression processing. Arbitrary position and width pulse (APWP) trains is a new class of SS signals which are expected to maintain advantages of the square pulse and the SS signals. Usual approach of SS signals application does not assume signal optimization. The novelty of the APWP approach is the optimization of the APWP train accounting of the signal transmission through system. In order to speed up the optimization iterations, a mathematical model of the APWP signal transmission through ultrasonic system is needed: instead of doing the iterations in real environment, those can be accomplished mathematically, using the system transmission model in order to sustain the experiment conditions. The development of the transmission model of the system is presented; the models are compared using the correlation properties. The results of the investigation are presented.
\end{abstract}

Index Terms-Ultrasonic imaging; ultrasound electronics; arbitrary position and width pulse signals; spread spectrum.

\section{INTRODUCTION}

Ultrasonic measurements are influenced by the excitation signal's quality and ultrasound transmission. The increase of the signal bandwidth or energy is improving the value of the imaging [1]. Energy enhancement is possible by increasing the amplitude of the probing signal or its duration [2]. Construction of the transducer and the capabilities of the electronics limit the probing signal amplitude [3]. Another approach would be to increase the duration of the exciting signal. This will reduce the envelope bandwidth of the signal and the sidelobes of the RF correlation function will be high. The obvious solution is to employ a spread spectrum signal [4]-[6] if wideband measurement channel is available: a processing gain allows for both resolution and accuracy improvement. Multiple spread spectrum techniques can be used: phase manipulated sequences [7], linear frequency modulation signals (chirp) [8]-[10], or arbitrary waveform signals [11], [12]. The phase-coded sequences do not offer flexibility in spectral content

Manuscript received 9 February, 2015; accepted 27 January, 2016

This research was funded by a project IN-SMART, grant No. VP1-3.1SMM-10-V-02-012. management [9], [13]. Arbitrary waveform excitation needs more complex electronics [11]. Chirp [8] excitation is considered the best choice but in its simplest form it generates high correlation sidelobes [7]. Trains of the arbitrary position and width pulses (APWP) [14] are a novel SS signal generation technique, offering both simple generation and adaptability to the transducer and the system frequency response. It requires multiple measurements to achieve the optimal response of the system. An optimization procedure can be carried out using the software, reducing the optimization time by several orders if the numerical model is available. The aim of the presented research is to develop such model, to check its validity and to evaluate its performance.

\section{CORRELATION PROPERTIES}

Taking the discrete version of the received ultrasonic signal $s t_{\mathrm{n}}$ and the reference signal $s r_{\mathrm{n}}$, representing the approximation of the signal transmitted, the crosscorrelation function (CCF) $x_{\mathrm{n}}$ is obtained as

$$
x_{m}=\sum_{n=1}^{N} s t_{n-m} \times s r_{n} .
$$

Such operation compresses the whole energy of the signal into one peak. This peak can be used to estimate the time of flight of the received signal. Thanks to the energy compression property, the SS signal which had energy spread over time, it now can be turned into single pulse. Several parameters of the $\mathrm{CCF}$ have to be discussed. If $\mathrm{CCF}$ is divided by the geometric mean of signal $s t_{\mathrm{n}}$ and the reference signal $s r_{\mathrm{n}}$ energies, correlation peak indicates the correlation coefficient

$$
C x_{m}=\max \left|x_{m} / \sqrt{\sum_{n=1}^{N}\left|s t_{n}\right|^{2} \sum_{n=1}^{N}\left|s r_{n}\right|^{2}}\right| .
$$

The correlation coefficient can be used to analyse the likeliness criteria of two signals.

The width of the RF mainlobe $\tau_{\mathrm{RF}}$ (Fig. 1) is representing the imaging resolution [15]. In discrete terms the mainlobe width $n \tau_{\mathrm{RF}}$ is obtained as

$$
m \tau_{R F}=\arg \left(C x_{m}=\left.0.5\right|_{m>m_{\text {peak }}}\right)-
$$




$$
-\arg \left(C x_{m}=\left.0.5\right|_{m<m_{\text {peak }}}\right)
$$

Mainlobe width of the envelope is representing the imaging resolution [16].

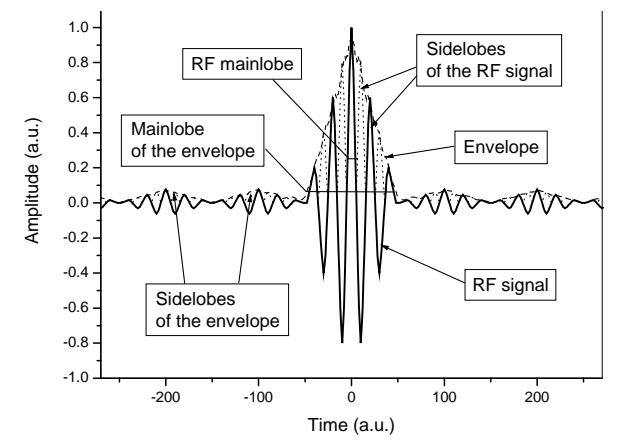

Fig. 1. Parameters of the signal correlation properties.

The ratio of the RF sidelobes peak to the mainlobe peak level (denoted by SLL $L_{R F}$ ) can be used to express the imaging contrast capability if the RF signal is used for imaging

$$
S L L_{R F}=20 \lg \frac{\left.\max \left[x_{m}\right]\right|_{m \neq \pm 0.5 n \tau_{R F}}}{\left.\max \left[x_{m}\right]\right|_{m= \pm 0.5 n \tau_{R F}}} .
$$

The envelope of the CCF is obtained by its Hilbert transform

$$
x e_{m}=\left|\operatorname{Hilb}\left(x_{m}\right)\right|=\left|\operatorname{IDFT}\left\{\operatorname{DFT}\left(x_{m}\right) \times H\left(f_{n}\right)\right\}\right| .
$$

The ratio of the envelope sidelobes to the mainlobe can be used to evaluate the conventional imaging contrast capability. The ratio of the RF sidelobes energy to the mainlobe energy (denoted by $\mathrm{SLE}_{\mathrm{RF}}$ ) can be used to evaluate the conventional imaging contrast capability

$$
S L E_{R F}=20 \lg \frac{\sum_{m=+0.5 n \tau_{R F}}^{N}\left(x_{m}\right)^{2}+\sum_{m=-N}^{-0.5 n \tau_{R F}}\left(x_{m}\right)^{2}}{\sum_{m=-0.5 n \tau_{R F}}^{+0.5 n \tau_{R F}}\left(x_{m}\right)^{2}} .
$$

Sidelobe to mainlobe ratio of the signal envelope, obtained using (5) represent the attainable contrast if signal envelope is used in imaging.

\section{APWP SIGNALS}

Figure 2 presents the excitation signal types which are easy to generate, i.e. where binary signals can be used for excitation. While pulse signal is the most common type in ultrasonic measurements, it has few drawbacks: all frequency components are concentrated in one time instant, therefore amplitude is high, which gives significant load to excitation electronics and ultrasonic transducer. Therefore the amount of energy delivered using the pulse excitation signal is limited. CW toneburst has the opposite property: the energy is high, but the bandwidth is narrow. Therefore it is not capable of high resolution imaging or measurement when reflections are close. The chirp signal is a SS signal that has both wide bandwidth and high energy. Yet, it has high sidelobes of RF and envelope of the correlation function. Phase manipulated sequences have good properties of the correlation envelope but are not able to account the response of the ultrasonic transducer and their RF sidelobes are also high (defined by the chip size).

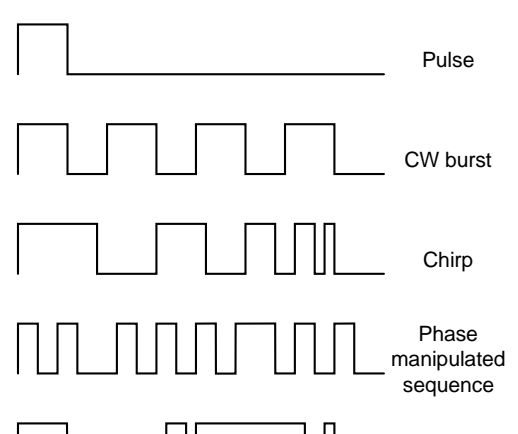

Fig. 2. Binary excitation signal types.

APWP signals offer the possibility to match the bandwidth of the ultrasonic transducer or even the whole signal transmission channel. Investigation presented in [13] has indicated that APWP signals can be optimized to produce correlation sidelobes that are lower than those of the pulse signal (Fig. 3).

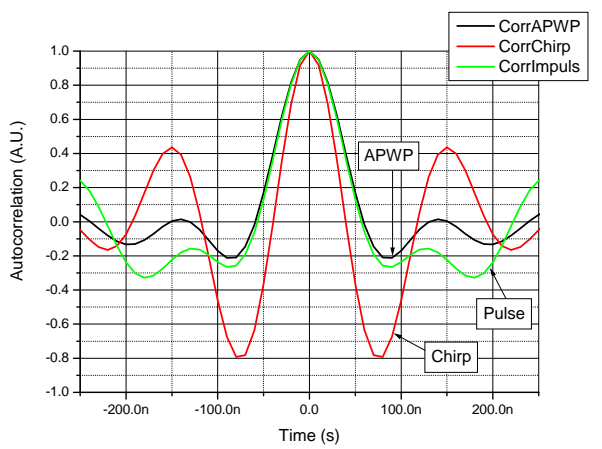

Fig. 3. Correlation function has been optimized for minimum SLL.

It can be clearly seen that APWP signal is able to provide the correlation properties comparable of even better than those of the pulse signal. Correlation sidelobes of the APWP are significantly lower than chirp signal, but it should be noted that result was at the expense of mainlobe width.

\section{SYSTEM MODEL}

Optimization of the APWP signals involves the system model. The candidate APWP signal is passed through the system model (Fig. 4) and the simulated output is treated as a real signal obtained at the reception end of the real ultrasonic system.

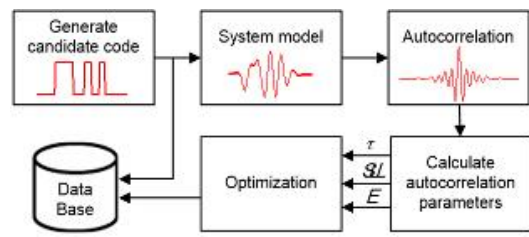

Fig. 4. The structure of the APWP signal optimization algorithm.

Then, this signal is used to obtain the auto-correlation function (AKF), submitted to analysis block to extract 
correlation properties discussed in section II and is stored in codes data base if it produces better correlation parameter than previous signal. The selected APWP codes can be used later in imaging applications [14] as optimal for a particular system-transducer combination.

\section{A. AC Response Based Model}

System model TT in [13], [14] was based on the frequency response of the system. It was determined by transmitting a code $s t_{n}$ of the chirp signal which was fed into ultrasonic pulser, submitted to ultrasonic transducer, propagated in water, reflected back from stainless steel reflector, was received by ultrasonic transducer, amplified, and registered using ADC as $s r_{\mathrm{n}}$ and then taking the DFTs

$$
T T(\omega)=\frac{D F T\left[s r_{n}\right]}{D F T\left[s t_{n}\right]} .
$$

Unfortunately, more new investigation (Fig. 5) revealed that such model is insufficient for system simulation.

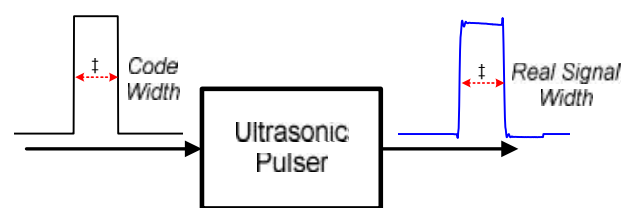

Fig. 5. Experimental setup for model adequacy investigation.

Ultrasonic pulser was fed with code defining the signal with variable duration. Output of the pulser was connected into ultrasonic transducer and reflection from same reflector in water registered. This real signal was compared to signal obtained using spectral model (7). The correlation coefficient (2) was used as the likelihood parameter (Fig. 6).

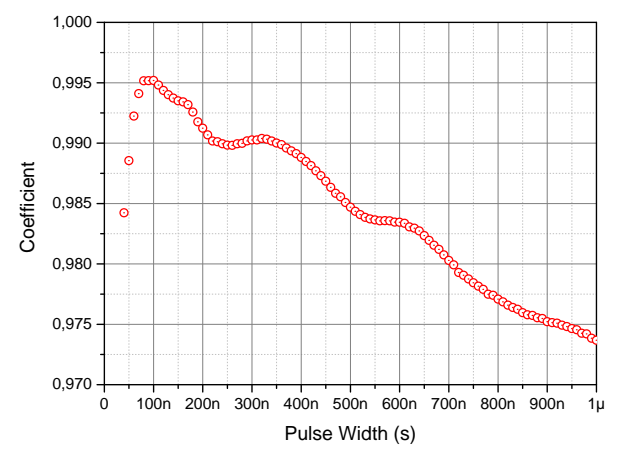

Fig. 6. The correlation coefficient vs. pulse duration for spectral model (7).

It can be seen that correlation coefficient was decreasing with the change of the pulse duration. It was assumed that the reason for the model correspondence reduction is the change in signal duration. To verify this assumption, duration of the code $\tau_{\text {code }}$ was compared against duration $\tau_{\text {real }}$ at the output

$$
\varepsilon\left(\tau_{i}\right)=\tau_{\text {code }}-\tau_{\text {real }} \text {. }
$$

Analysis of Fig. 7 reveals that a better model, capable to reflect the pulse width was needed.

\section{B. Convolutional Model}

The convolutional model (see explanatory diagrams in Fig. 8) was suggested to replace the spectral model.

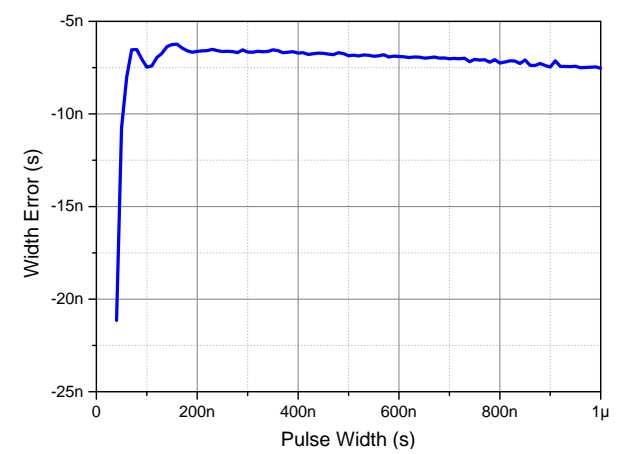

Fig. 7. Deviation of the pulse duration at pulser output from the set value.

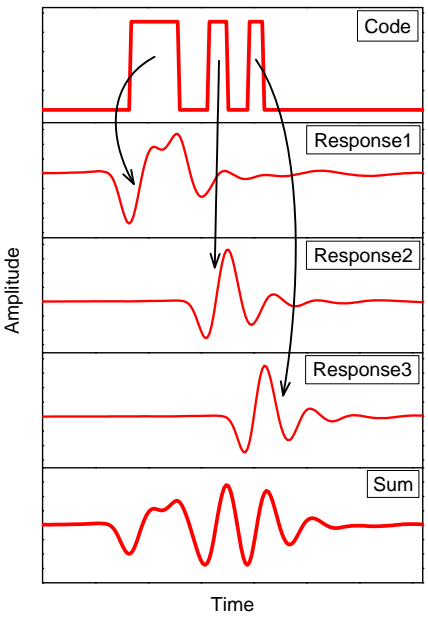

Fig. 8. Explanation of the convolutional model operation.

The signals recorded at receiving ultrasonic transducer in the experiment presented in Fig. 5 were used to construct the response to the supplied code. The code was submitted to duration analysis which determines the duration of the current APWP pulse. Then the previously (corresponding duration) recorded signal was convolved with the responses of the other pulses.

The convolutional model was compared against the spectral model using real signals, recorded using several APWP codes. The correlation coefficient (2) between the simulated and the real signals was obtained (Fig. 9).

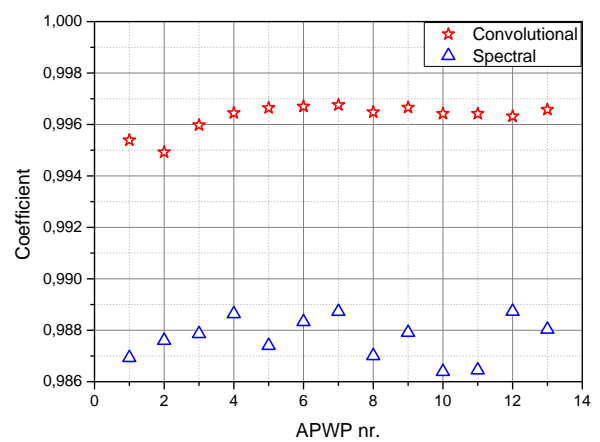

Fig. 9. Correlation coefficient between simulated and real signals.

It can be seen that the convolution model is producing a closer correlation to the real signal when compared to the spectral model. The lowest correlation coefficient for the convolutional model is 0.995 and 0.986 for spectral model. The highest coefficients are 0.997 (convolutional) and 0.989 (spectral). Even spread of the coefficients for convolutional is twice lower.

The same signals were compared by mainlobe width using (8). The results of the errors when compared to the 
real signal are presented in Fig. 10. It can be seen that the convolutional model provides a better CCF mainlobe duration match with the real signal. The maximum deviation of mainlobe duration for convolutional model is $1.8 \mathrm{~ns}$ and $6.3 \mathrm{~ns}$ for spectral model. The mean error is almost zero for the convolutional model but reaches $3 \mathrm{~ns}$ for the spectral model. The standard deviation of the error in the mainlobe width simulation was small, yet significantly higher for spectral model $(1.2 \mathrm{~ns})$ compared to convolutional model (0.3 ns).

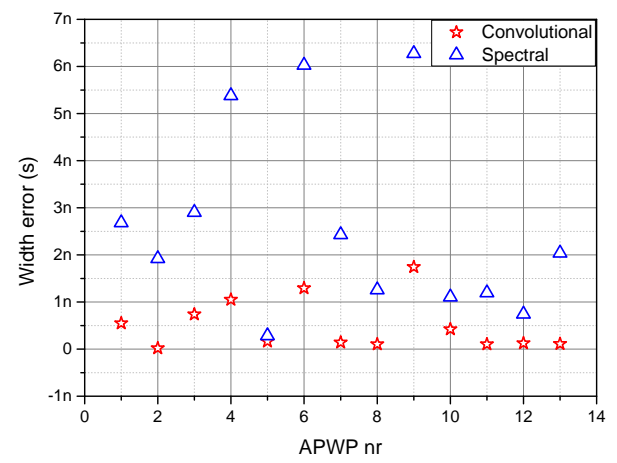

Fig. 10. Mainlobe width error of simulated signals for APWP codes.

The performance of the developed models was compared for simulated sidelobes level which was obtained using equation (4). Sidelobe level of the signals was compared versus the similar parameter, obtained for real signals. Results of the simulated sidelobe level deviation from one obtained using the real signals are presented in Fig. 11.

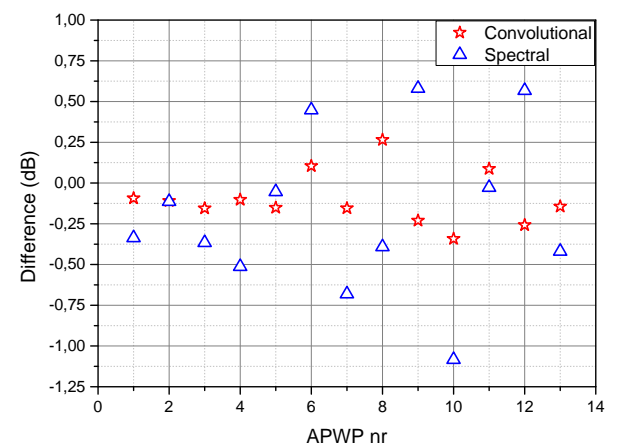

Fig. 11. Correlation sidelobe level difference for various APWP codes.

Analysis of Fig.11 reveals that models' performance in correlation sidelobe level simulation is similar. Yet, the spectral model has a slightly larger deviation of $\mathrm{CCF}$ sidelobe level (maximum error $0.6 \mathrm{~dB}$ ). The performance of the convolutional model is slightly better (maximum error $0.3 \mathrm{~dB})$.

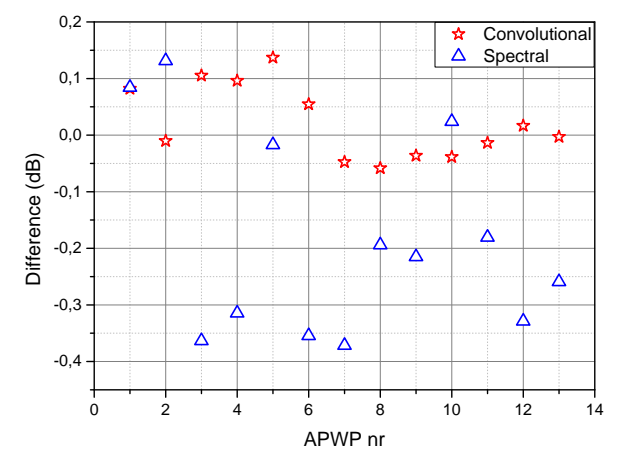

Fig. 12. Correlation sidelobe energy difference for various APWP codes.
The sidelobe energy analysis used (6). Results are presented in Fig. 12.

Analysis of Fig. 12 indicates similar performance of the models in correlation sidelobe energy approximation. Nevertheless, convolutional model has a slightly better CCF sidelobe energy match to the real signal $(0.14 \mathrm{~dB}$ convolutional vs. $0.38 \mathrm{~dB}$ for spectral model).

\section{CONCLUSIONS}

Two ultrasonic system transmission models to be used in APWP signals optimization were developed. The investigation revealed that both models are adequate to be used in system transmission simulation. The correlation properties of the model comparison against the real signals revealed that the spectral model has lower performance compared to the convolutional one.

\section{REFERENCES}

[1] M. Kulokas, R. Kazys, L. Mazeika, "Non-destructive evaluation of green ceramic body density by ultrasonic technique", Elektronika ir Elektrotechnika, no. 5, pp. 71-76, 2011.

[2] S. Kageyama, H. Hasegawa, H. Kanai, "Increasing bandwidth of ultrasound radio frequency echoes using Wiener filter for improvement of accuracy in measurement of intima-media thickness", Japanese journal of applied physics, vol. 52, no. 7, pp. 1-6, 2013.

[3] I. Morkvenaite-Vilkonciene, D. Virzonis, G. Vanagas, V. Krikscikas, "Operating point of capacitive micromachined ultrasonic transducers with sub-structural elements", Elektronika ir Elektrotechnika, vol. 18 no. 9 , pp. $43-46,2012$

[4] L. R. Welch, M. D. Fox, "Practical spread spectrum pulse compression for ultrasonic tissue imaging", IEEE Trans. Ultrason. Ferroelect. Freq. Control, vol. 45, no. 2, pp. 349-355, 1998.

[5] Z. Haichong, et al., "Simultaneous multispectral coded excitation using periodic and unipolar $\mathrm{m}$-sequences for photoacoustic imaging", Conf. Photons Plus Ultrasound - Imaging and Sensing Location, 2013.

[6] C. Xiaodong, et al., "Increasing average power in medical ultrasonic endoscope imaging system by coded excitation", International Conf. Optical Instruments and Technology, 2009.

[7] A. Nowicki, I. Trots, P. A. Lewin, et. al., "Influence of the ultrasound transducer bandwidth on selection of the complementary Golay bit code length", Ultrasonics, vol. 47, pp. 64-73, 2007.

[8] M. Pollakowski, H. Ermert, "Chirp signal matching and signal power optimization in pulse-echo mode ultrasonic nondestructive testing", IEEE Trans. Ultrason., Ferroelectr., Freq. Control, vol. 41, no. 5 , pp. 655-659, 1994.

[9] A. Kasaeifard, J. Saniie, "A successive parameter estimation algorithm for chirplet signal decomposition", IEEE Int. ultrasonics symposium (IUS), pp. 1465-1468, 2012.

[10] T. Virolainen, J. Eskelinen, E. Haeggstrom, "Frequency domain low time-bandwidth product chirp synthesis for pulse compression side lobe reduction", IEEE Int. ultrasonics symposium (IUS), pp. 15261528,2012

[11] D. Cowell, S. Freear, "Quinary excitation method for pulse compression ultrasound measurements", Ultrasonics, vol. 48, pp. 98 $108,2008$.

[12] Z. Haichong, et al., "Simultaneous multispectral coded excitation using periodic and unipolar M-sequences for photoacoustic imaging", Conf. Photons Plus Ultrasound - Imaging and Sensing Location, 2013.

[13] L. Svilainis, et al., "Application of arbitrary position and width pulse train signals in ultrasonic imaging", in 2014 Proc. ECNDT Conf. Prague, pp. 1-9.

[14] L. Svilainis, et al., "Application of arbitrary position and width pulse trains signals in ultrasonic imaging: correlation performance study", Elektronika ir Elektrotechnika, vol. 19, no. 3, pp. 57-60, 2013.

[15] R. Raisutis, R. Kazys, L. Mazeika, "Ultrasonic thickness measurement of multilayered aluminum foam precursor material", IEEE Trans. Instrum. Meas, vol. 57, no. 12, pp. 2846-2855, 2008.

[16] T. Misaridis, J. A. Jensen, "Use of modulated excitation signals in medical ultrasound. Part II: design and performance for medical imaging applications", IEEE Trans. Ultrason. Ferroelect. Freq. Control, vol. 52, no. 2, pp. 192-207, 2005. 